\title{
Natural convection in a triangular enclosure due to non-uniform cooling on top
}

\author{
Suvash C. Saha ${ }^{1} \quad$ Y. T. Gu ${ }^{2}$
}

(Received 8 January 2012; revised 11 April 2012)

\begin{abstract}
Natural convection in a triangular enclosure subject to non-uniform cooling at the inclined surfaces and uniform heating at the base is investigated numerically. The numerical simulations of the unsteady flows over a range of Rayleigh numbers and aspect ratios are carried out using the Finite Volume Method. Since the upper surface is cooled and the bottom surface is heated, the air flow in the enclosure is potentially unstable to Rayleigh-Benard instability. Based on the numerical results, the transient flow development in the enclosure is classified into three distinct stages: an early stage, a transitional stage, and a steady stage. In addition, the flow inside the enclosure strongly depends on the governing parameters; Rayleigh number and aspect ratio. The asymmetric behaviour of the flow about the geometric centre line is discussed in detailed. The heat transfer through the roof and the ceiling as a form of Nusselt number is also reported.
\end{abstract}

http://journal.austms.org.au/ojs/index.php/ANZIAMJ/article/view/5088 gives this article, (c) Austral. Mathematical Soc. 2012. Published April 26, 2012. ISSN 1446-8735. (Print two pages per sheet of paper.) Copies of this article must not be made otherwise available on the internet; instead link directly to this URL for this article. 


\section{Contents}

1 Introduction

C54

2 Governing equations and the system

C56

3 Numerical technique

C58

4 Results and discussion

C58

4.1 Overall flow development . . . . . . . . . . . . . C59

4.1.1 Start-up stage flow . . . . . . . . . . . . C59

4.1.2 Transitional stage flow . . . . . . . . . . . . C60

4.1 .3 Steady stage flow . . . . . . . . . . . . . C62

4.2 Effect of Rayleigh numbers on flow development . . . . . C62

4.3 Effect of aspect ratio on flow development . . . . . . . C64

4.4 Observation of the asymmetric flow behavior . . . . . . C65

5 Conclusion

C65

References

C67

\section{Introduction}

Natural convection in the triangular shaped enclosures has received a considerable attention recently due to its direct application in various engineering fields such as building and thermal insulation systems, solar engineering applications, geophysical fluid mechanics. Kamiyo et al. [1] and Saha and Khan [2] extensively reviewed work related to natural convection in the triangular cavity. The majority of existing research is focused on the numerical simulations of steady state flows and heat transfer $[3,4,5]$. Some research has been conducted for the transient flow response $[6,7,8,9,10]$. Poulikakos and Bejan [6] at first studied the scaling analysis of the transient flow inside an 
attic space for the case of night time boundary condition with the assumption that the flow is symmetric about the geometric centre plane with a very small aspect ratio. Saha and co-workers [8, 9, 10] performed a modified scaling analysis for both heating and cooling conditions on the inclined surfaces of the attic. They validated their scaling results with the numerical results for a wide range of parameters.

Previously, the flow inside the enclosure was considered as symmetric about the symmetric centre plane. However, Holtzman et al. [11] first observed the asymmetric flow structures in an isosceles triangular enclosure due to the formation of a pitchfork bifurcation for the night time cooling condition. A similar flow structure was also observed by Saha et al. [12] for periodic thermal forcing condition on the sloping walls of the attic space.

In the present study a non-uniform surface temperature on the sloping wall is considered where the bottom surface is uniformly heated. We expect that the temperature on roofs of the attic shaped buildings may not be uniformly distributed. The effects of the aspect ratios and Rayleigh numbers on the fluid flow and heat transfer are discussed in detail. The formation of a pitchfork bifurcation of the fluid flow about the symmetric centre line is observed. The numerical simulations reveal that the flow development inside the enclosure undergoes several stages; for example, an early stage, a transition stage and a steady stage. The numerical results reveal that for lower Rayleigh numbers, the flow is symmetric; however, it becomes asymmetric when the Rayleigh number exceeds a critical value. The influence of natural convection on heat transfer rates is also important to understand the systems with various applications. The detailed analysis of heat transfer is carried out with average Nusselt number and such analysis on the role of natural convection in heat transfer effects in triangular cavities is reported in this study. 


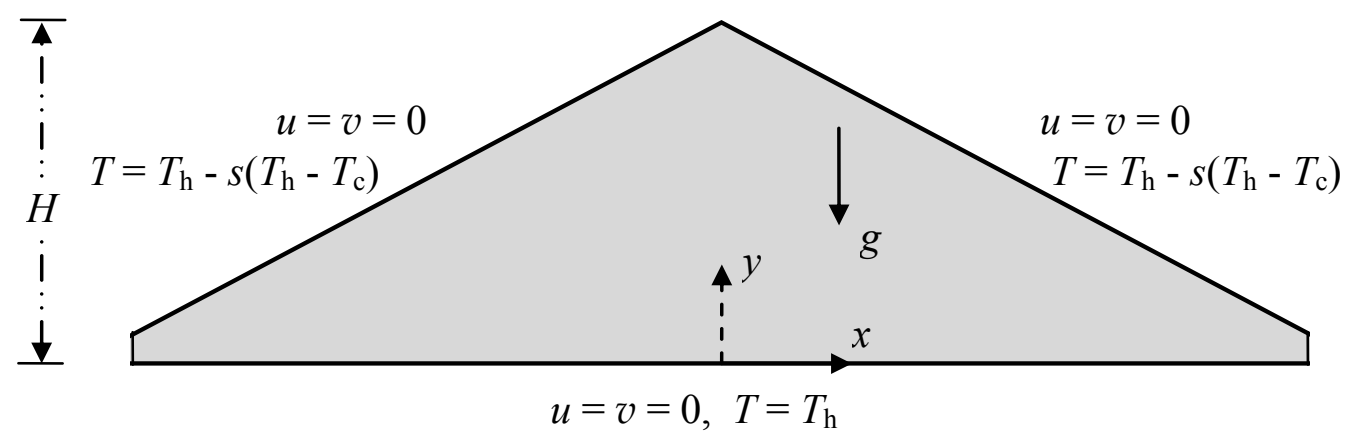

Figure 1: Schematic of the geometry and the coordinate system.

\section{Governing equations and the system}

Under consideration is the unsteady flow behaviour resulting from a quiescent, isothermal Newtonian fluid with Prandtl number $\operatorname{Pr}=0.72$ in a two dimensional triangular enclosure of height $\mathrm{H}=0.14 \mathrm{~m}$ and horizontal length $2 \mathrm{l}(\mathrm{l}=0.28 \mathrm{~m}, 0.56 \mathrm{~m}$ and $1.40 \mathrm{~m}$ for three different aspect ratios) by imposing a linear cooling temperature condition on both inclined walls, as shown in Figure 1. The horizontal bottom surface is uniformly heated at temperature $T_{h}(K)$. The initial fluid temperature is cold $T_{c}\left(T_{c}<T_{h}\right)(K)$. The flow is assumed as laminar. Moreover, in order to avoid the singularities at the tips in the numerical simulation, the tips are cut off by $5 \%$ and at the cutting points (refer to Figure 1) rigid non-slip and adiabatic vertical walls are assumed. We anticipate that this modification of the geometry would not alter the overall flow development significantly.

The evolution of the temperature and flow fields in the triangular enclosure following the start-up, which is dominated by natural convection, is described by the following set of governing equations, for which the Boussinesq assumption 
has been made

$$
\begin{aligned}
& \frac{\partial u}{\partial x}+\frac{\partial v}{\partial y}=0 \\
& \frac{\partial u}{\partial t}+u \frac{\partial u}{\partial x}+v \frac{\partial u}{\partial y}=-\frac{1}{\rho} \frac{\partial p}{\partial x}+v\left(\frac{\partial^{2} u}{\partial x^{2}}+\frac{\partial^{2} u}{\partial y^{2}}\right) \\
& \frac{\partial v}{\partial t}+u \frac{\partial v}{\partial x}+v \frac{\partial v}{\partial y}=-\frac{1}{\rho} \frac{\partial p}{\partial y}+v\left(\frac{\partial^{2} v}{\partial x^{2}}+\frac{\partial^{2} v}{\partial y^{2}}\right)+g \beta\left(T-T_{c}\right) \\
& \frac{\partial T}{\partial t}+u \frac{\partial T}{\partial x}+v \frac{\partial T}{\partial y}=\kappa\left(\frac{\partial^{2} T}{\partial x^{2}}+\frac{\partial^{2} T}{\partial y^{2}}\right)
\end{aligned}
$$

where $u$ and $v$ are the $\boldsymbol{x}$-direction and $\boldsymbol{y}$-direction velocity components, $\boldsymbol{t}$ the time, $p$ the pressure, $T$ the temperature, $g$ the acceleration due to gravity and $\beta, v$ and $\kappa$ are the thermal expansion coefficient, kinematic viscosity and thermal diffusivity of the fluid at temperature $T_{c}$, respectively.

The initial and boundary conditions are:

- initially the fluid is quiescent and isothermal at temperature $T_{c}$;

- on the sloping walls a rigid non-slip and non-uniform temperature conditions are applied with a linear temperature function, $T=T_{h}-$ $s\left(T_{h}-T_{c}\right)$, where $s$ is the length of the inclined surface;

- the bottom horizontal wall is maintained with a constant uniform heating and rigid non-slip;

- at the cutting points of the bottom tips, rigid non-slip and adiabatic vertical walls are assumed.

The flow development is determined by three governing parameters; the Rayleigh number, Ra, the Prandtl number, Pr, and the aspect ratio, A. They are defined respectively as

$$
\mathrm{Ra}=\frac{g \beta \Delta T \mathrm{H}^{3}}{\kappa \nu}, \quad \operatorname{Pr}=\frac{\nu}{\kappa} \quad \text { and } \quad A=\frac{H}{l},
$$


where $\Delta T$ is the temperature difference $\left(T_{h}-T_{c}\right)$. The average Nusselt number on the surfaces is also defined as

$$
\mathrm{Nu}=\frac{h_{\mathrm{eff}} \mathrm{H}}{k},
$$

where the heat transfer coefficient

$$
h_{\mathrm{eff}}=\frac{\mathrm{q}^{\prime \prime}}{\Delta \mathrm{T}}
$$

with $q^{\prime \prime}$ being the total surface heat flux.

\section{$3 \quad$ Numerical technique}

Two dimensional numerical simulations are carried out in this study. For this purpose, an isosceles triangular domain is considered, and a Cartesian coordinate system is adopted with the origin located at the centre of the base, the $x$-direction pointing horizontally, and the $y$-direction pointing vertically. The initial and boundary conditions for the numerical simulations are also specified. That is, the air in the enclosure is initially quiescent and isothermal with a uniform temperature of $T_{c}$. At the time $t=0$, the two inclined surfaces are linearly cooled and the base of the enclosure is uniformly heated. All three surfaces of the enclosure are assumed rigid and no slip. The detailed numerical procedure and extensive grid and time step dependent test were reported by Saha and co-workers [8, 9, 10].

\section{Results and discussion}

The transient development of the flow in the isosceles triangular enclosure in response to the uniform heating through the horizontal and non-uniform cooling through the inclined surfaces is described based on the numerical 
simulations. Other features of the transient flow and heat transfer, as well as their dependency on the Rayleigh number and aspect ratio, are further explored.

\subsection{Overall flow development}

Here, the overall features of the flow development in the isosceles triangular enclosure are described based on the Rayleigh number of $\mathrm{Ra}=10^{6}$ and aspect ratio of $A=0.5$. The discussion of the different stages of the development of the transient flow is given below.

\subsubsection{Start-up stage flow}

As mentioned previously, the air in the isosceles triangular enclosure is initially isothermal and stationary. At the start-up of the numerical simulation, nonisothermal conditions are established over the inclined surfaces, which linearly cool the enclosure through the inclined surfaces and uniformly heat the enclosure through the base. As a consequence, thermal boundary layers develop along all the interior surfaces. Among them, the thermal boundary layer adjacent to the base is a heating thermal boundary layer and those which are adjacent to the inclined surfaces are a bit complex. The top portion of the inclined surface generates a cooling thermal boundary layer and the bottom portion generates a heating thermal boundary layer. Figure 2 shows the growth of these thermal boundary layers with time, and which shows the isotherms (Figure 2a,c) and contours of stream functions (Figure 2b,d) at two different times following the start-up.

The isotherms in Figure 2 show that, despite the growth of the thermal boundary layers inside the enclosure, the air in the core remains isothermal at the initial temperature $(290 \mathrm{~K})$ at the early stage. Since the bottom portions of the inclined walls are hot and the top portion is cold, the hot fluid from the bottom moves through the boundary layer to the top. On the other hand, 

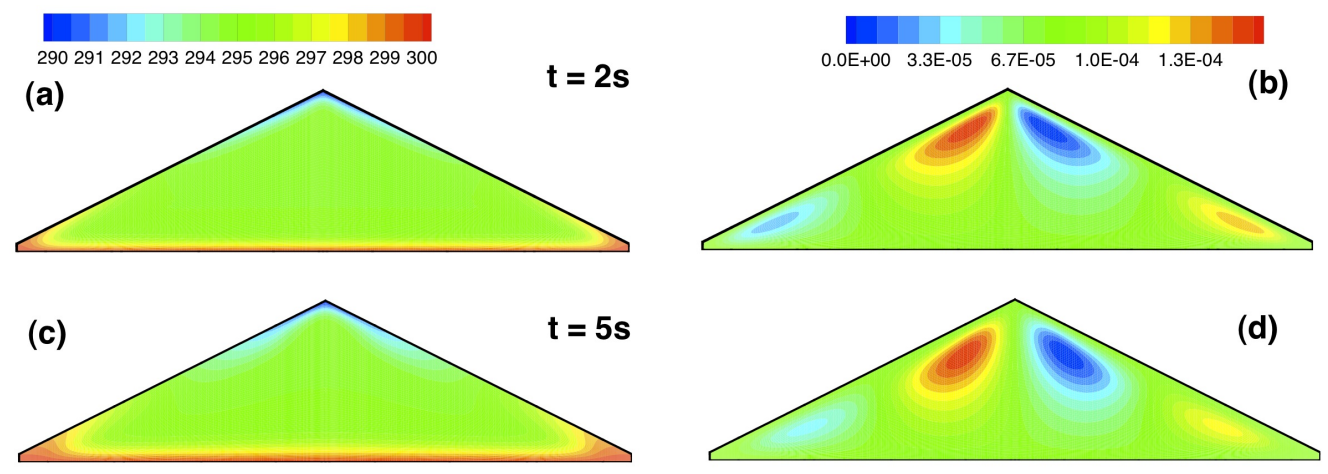

Figure 2: Isotherms (left) and streamlines (right) for $\mathrm{Ra}=10^{6}$ and $\mathrm{A}=0.5$ at the start up stage

the cold air from the top moves towards the bottom through the boundary layer adjacent to the inclined walls. The hot and cold air meets at around the mid portion of the inclined walls and discharges into the core. Adjacent to the bottom surface, there is another hot boundary layer that starts to grow since the initiation of the thermal forcing. The comparison between the isotherms at the two different times indicates the increase with time of the thicknesses of all thermal boundary layers toward the core. The contours of the stream functions at this stage show four weak rotating cells, two of them are on each side of the geometric symmetry plane, which are formed due to entrainment of the cooling/heating thermal boundary layers. The rotation of the symmetric cells about the mid plane has opposite direction. The flow is symmetric at this stage.

\subsubsection{Transitional stage flow}

The flow at the transitional stage is characterised by the appearance of convective instabilities as a form of rising and sinking plumes. The horizontal thermal boundary layer with warm air underneath cool air is formed due to 

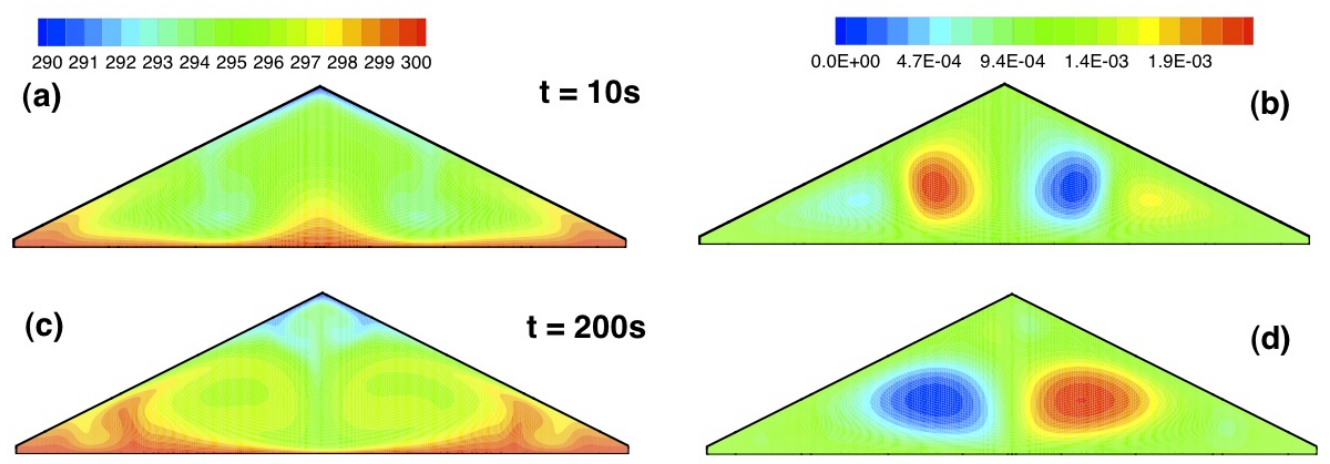

Figure 3: Isotherms (left) and streamlines (right) for $\mathrm{Ra}=10^{6}$ and $\mathrm{A}=0.5$ at the transitional stage.

heating the bottom surface, which is potentially unstable to Rayleigh-Benard instabilities. The heating thermal boundary layer becomes unstable as soon as the critical condition is met. Figure 3 shows the isotherms (left) and streamlines (right) for two different times in this regime. Since the air with two different temperature meets near around the mid portion of the inclined surfaces through the boundary layer, it has no choice but to move downward. As a result, the hot air plume travels towards the centre of the enclosure and the cold air plumes travels from two inclined surfaces to the bottom surface. A mixing of hot and cold air starts to happen. As time progress the flow becomes stronger and eventually asymmetric as a result of pitchfork bifurcation. We observe that the flow oscillates for quite a long time before it becomes steady state. During the time of oscillation, the bifurcation repeats left and right about the symmetric centre line. Due to the occurrence of the convective instabilities, the four cell flow structures observed at the early stage of the flow development are broken into multiple cells, as shown by the contours of the stream functions in Figure 3. 
290291292293294295296297298299300

(a)

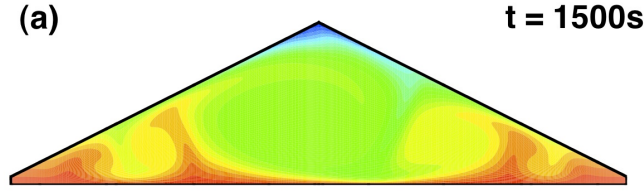

$0.0 \mathrm{E}+00 \quad 5.8 \mathrm{E}-04 \quad 1.2 \mathrm{E}-03 \quad 1.8 \mathrm{E}-03 \quad 2.3 \mathrm{E}-03 \quad 2.9 \mathrm{E}-03$

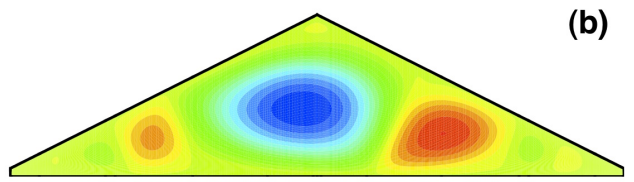

Figure 4: Isotherms (left) and streamlines (right) for $\mathrm{Ra}=10^{6}$ and $\mathrm{A}=0.5$ at the steady stage.

\subsubsection{Steady stage flow}

Figure 4 shows the isotherms and streamlines at the steady stage. In the previous regime (Section 4.1.2), if the Rayleigh number exceeds a critical value, the flow is observed to undergo a supercritical pitchfork bifurcation, in which case one of two possible mirror image asymmetric solutions are obtained [11]. We found in the previous regime that the dominant plume, which is formed at the apex oscillates about the geometric symmetry plane for quite a long time (about 18 mins). However, before getting steady state it moves to right side of the enclosure in this case. At the steady stage, Figure 4 shows that a large convecting cell forms in the centre of the enclosure for the aspect ratio considered here. The occurrence of the convective instabilities results in a number of small secondary convective cells in addition of the large dominant cell in the enclosure.

\subsection{Effect of Rayleigh numbers on flow development}

The present simulations cover a range of Rayleigh numbers, $5.0 \times 10^{4} \leqslant \mathrm{Ra} \leqslant$ $1.0 \times 10^{6}$. Different features of the transient flows are observed over the range of Rayleigh numbers. The isotherms and the corresponding streamlines for different Rayleigh numbers are shown in Figure 5 for $A=0.5$. From the comparisons of the numerical results for the different Rayleigh numbers, we 

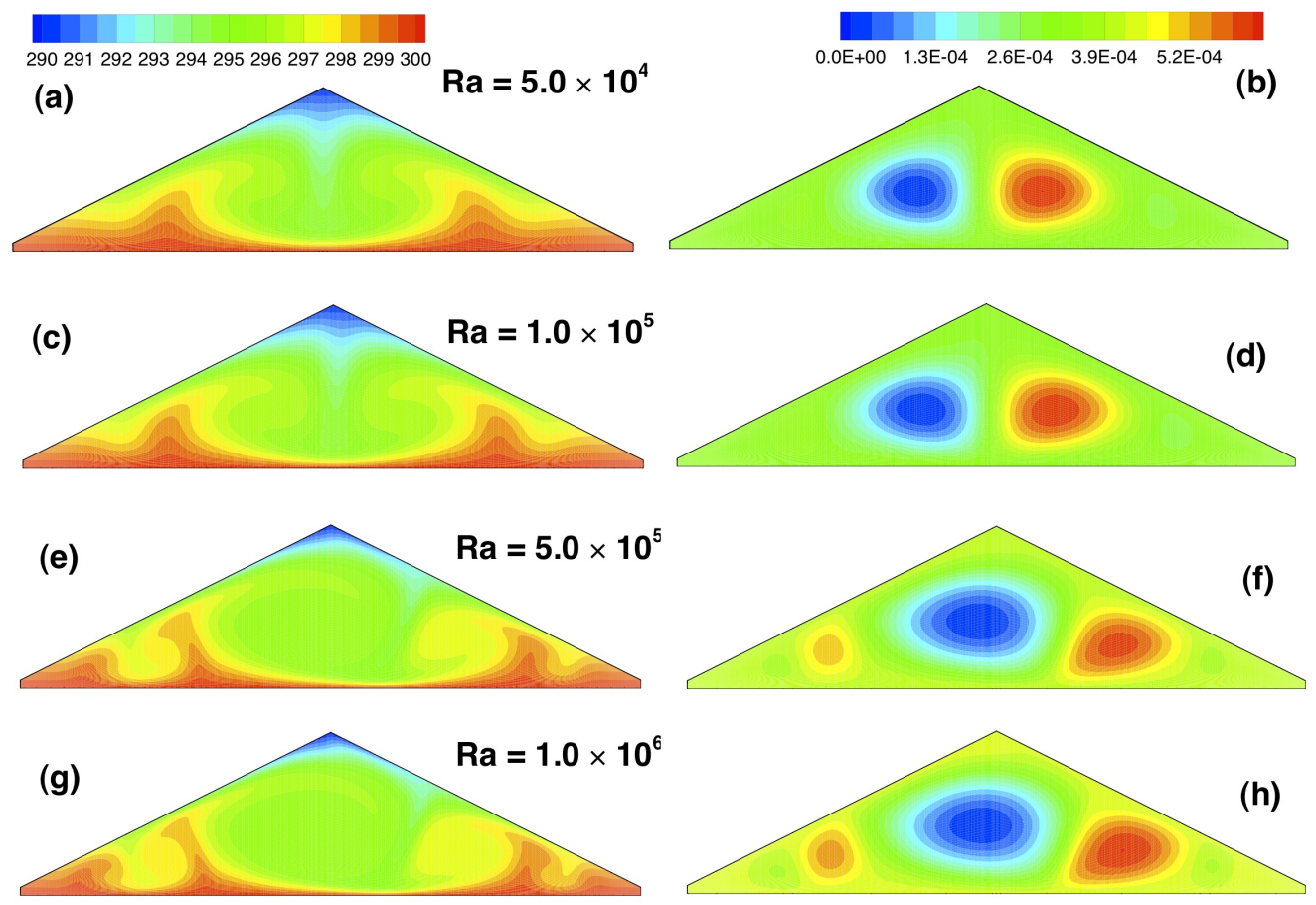

Figure 5: Steady state flow for different Rayleigh numbers for a fixed $A=0.5$ : (left) isotherms (right) contours of stream functions

notice certain variations of the flow features. Firstly, the convective flow instabilities are observed even at the lowest Rayleigh number case. With the increase of the Rayleigh number, the instabilities exaggerate and the associated wave number increases. Secondly, for lower Rayleigh numbers $\left(\mathrm{Ra}=5.0 \times 10^{4}\right.$ and $\left.1.0 \times 10^{5}\right)$, the flow is nearly symmetric. However, the flow becomes asymmetric for higher Rayleigh numbers $\left(\mathrm{Ra}=5.0 \times 10^{5}\right.$ and $\left.1.0 \times 10^{6}\right)$. Finally, when the flow is symmetric, two counter rotating primary cells dominant the flow. A strong primary cell dominates the flow when the flow becomes asymmetric for higher Rayleigh numbers. 

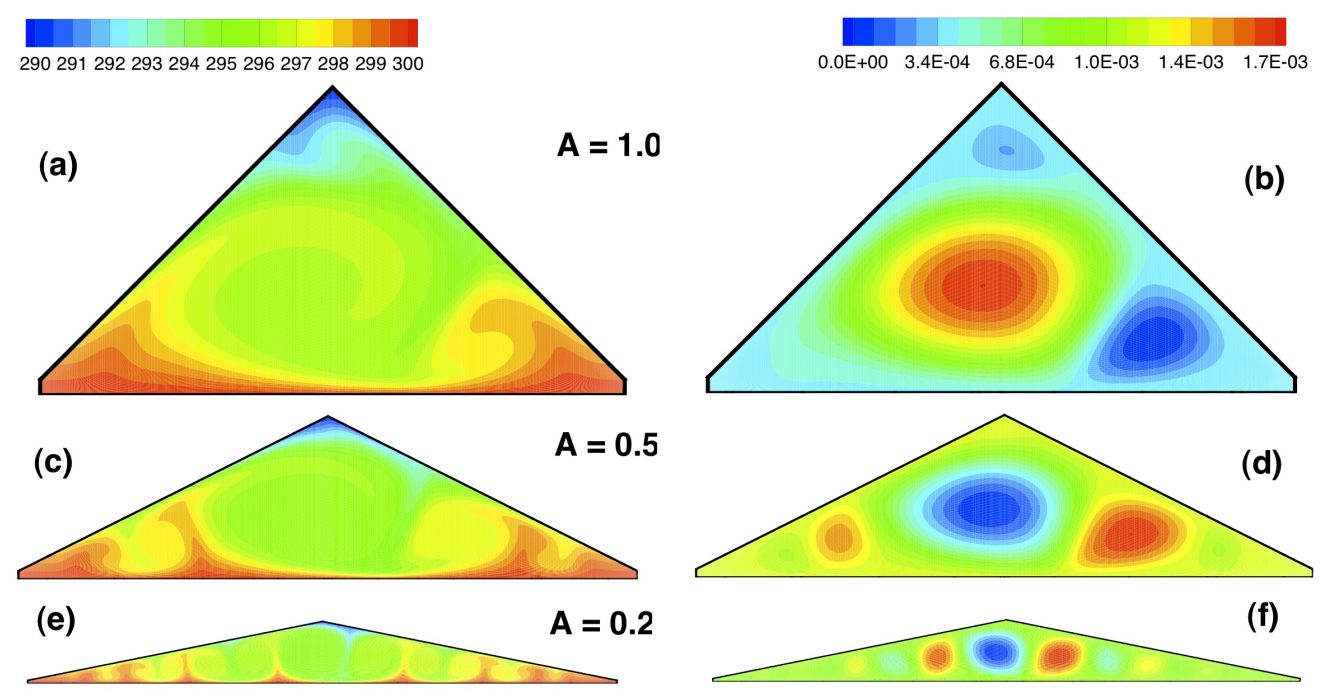

Figure 6: Steady state flow for different aspect ratios for a fixed Ra $=10^{6}$ : (left) isotherms and (right) contours of stream functions.

\subsection{Effect of aspect ratio on flow development}

Figure 6 shows the isotherms and contours of the stream functions for different aspect ratios with fixed Rayleigh number, $\mathrm{Ra}=1.0 \times 10^{6}$. Similar to the variation of Rayleigh numbers, comparisons of the numerical results for different aspect ratios also reveal certain variations of the flow features. Firstly, the convective flow instabilities are observed for all aspect ratios. Secondly, the flow is asymmetric along the geometric centre line for every aspect ratio. Thirdly, as aspect ratio decreases the number of convecting cells increases. 


\subsection{Observation of the asymmetric flow behavior}

Holtzman et al. [11] and Saha et al. [12] claimed that the transition of the flow from symmetry to asymmetry is due to the occurrence of a pitchfork bifurcation. In this study the pitchfork bifurcation is observed in more detail. Two time series of the calculated temperatures at two locations within the heating thermal boundary layer for $\mathrm{Ra}=1.0 \times 10^{6}$ are shown in Figure 7(a). Two locations are selected as $A(-0.06 \mathrm{~m}, 0.07 \mathrm{~m})$ and $B(0.06 \mathrm{~m}, 0.07 \mathrm{~m})$ which are symmetric about the geometric symmetry plane. Figure 7 (a) shows that the temperature at the two locations are the same for a significant period of time. This indicates that the flow is symmetric about the geometric symmetry line during this period. However, the two temperature time series start to diverge at about $200 \mathrm{~s}$ which is seen in Figure 7(b) where the time history of the temperature difference between these two locations is plotted. That means the pitchfork bifurcation has occurred and the transition of the flow to asymmetry is established. After the initiation of the pitchfork bifurcation the flow oscillates. These oscillations last a significant time before getting steady state. The pitchfork bifurcation has also been investigated by calculating the heat transfer of the left and the right inclined surfaces of the enclosure. Figure 7(c) and Figure 7(d) plot the time series of the average Nusselt numbers on the two inclined surfaces and the variation of the Nusselt number between two surfaces respectively. The calculated results exhibit similar features as for the case of calculation of temperature time series of two points.

\section{Conclusion}

The natural convection flow in an isosceles triangular enclosure subject to nonuniform cooling from the inclined surfaces and uniform heating from the base is investigated numerically. The numerical simulation is performed using a Finite Volume Method for a range of aspect ratios $(0.2<A<1.0)$ and Rayleigh numbers $\left(5.0 \times 10^{4}<\mathrm{Ra}<1.0 \times 10^{6}\right)$. The numerical simulations reveal that 

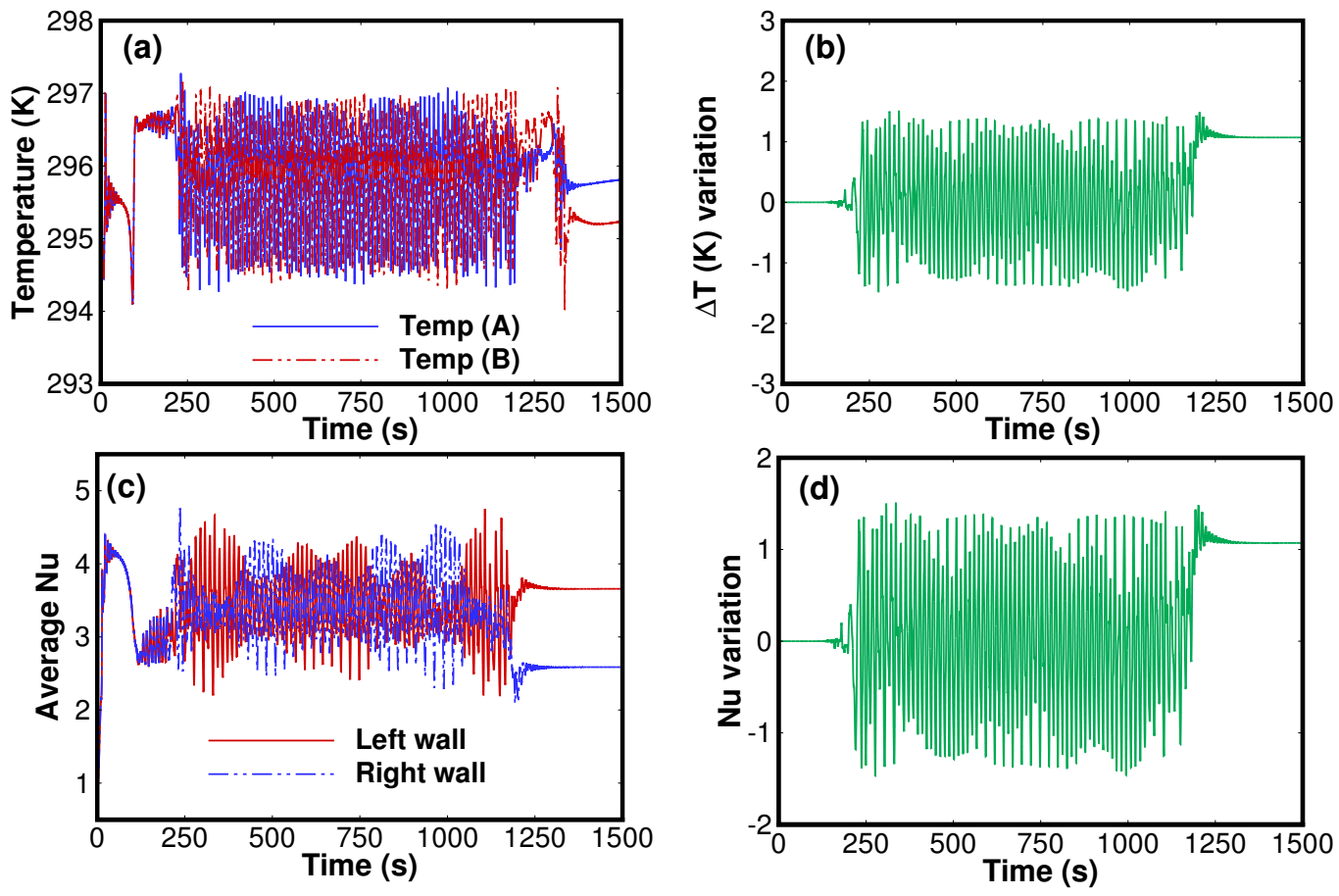

Figure 7: Observation of the occurrence of the pitchfork bifurcation $(A=$ $\left.0.5, \mathrm{Ra}=10^{6}\right)$.

the transient flow development inside the enclosure can be classified into three distinct stages: an early stage, a transitional stage, and a steady stage. The early stage flow is characterised by the growth of thermal boundary layers adjacent to all the interior surfaces and the initiation of primary circulations. The transitional stage flow is characterised by the appearance of convective instabilities as the form of rising and sinking plumes and the formation of cellular flow structures. The steady state flow is characterised by symmetric flows about the geometric symmetry plane for lower Rayleigh numbers and by the pitchfork bifurcation when the Rayleigh number is higher. The transition of the flow from symmetry to asymmetry due to the pitchfork bifurcation is examined in detail in this study. Finally, the features of the heat transfer 
through the enclosure are examined.

\section{References}

[1] O. M. Kamiyo, D. Angeli, G. S. Barozzi, M. W. Collins, V. O. S. Olunloyo, S. O. Talabi, A comprehensive review of natural convection in triangular enclosures, Applied Mechanics Reviews, 63, 060801. doi:10.1115/1.4004290 C54

[2] S. C. Saha, M. M. K. Khan, A review of natural convection and heat transfer in attic-shaped space, Energy and Buildings, 43, 2564-2571. doi:10.1016/j.enbuild.2011.06.020 C54

[3] V. A. Akinsete, T. A. Coleman, Heat transfer by steady laminar free convection in triangular enclosures, Int. J. Heat Mass Trans., 25, 1982, 991-998. doi:10.1016/0017-9310(82)90074-6 C54

[4] E. M. del Campo, M. Sen, E. Ramos, Analysis of laminar natural convection in a triangular enclosure, Numer. Heat Transfer, 13, 1988, 353-372. doi:10.1080/10407788808913618 C54

[5] H. Asan, L. Namli, Numerical simulation of buoyant flow in a roof of triangular cross-section under winter day boundary conditions, Energy and Buildings 33, 2001, 753-757. doi:10.1016/S0378-7788(01)00063-9 $\mathrm{C} 54$

[6] D. Poulikakos, A. Bejan, The fluid dynamics of an attic space, J. Fluid Mech. 131, 1983, 251-269. doi:10.1017/S0022112083001317 C54

[7] C. Lei, S. W. Armfield, J. C. Patterson, Unsteady natural convection in a water-filled isosceles triangular enclosure heated from below, Int. J. Heat Mass Trans. 51, 2008, 2637-2650. doi:10.1016/j.ijheatmasstransfer.2007.09.036 C54 
[8] S. C. Saha, J. C. Patterson, C. Lei, Natural convection in attic-shaped spaces subject to sudden and ramp heating boundary conditions, Heat Mass Transfer, 46, 2010, 1432-1181. doi:10.1007/s00231-010-0607-5 C54, C55, C58

[9] S. C. Saha, Unsteady natural convection in a triangular enclosure under isothermal heating, Energy and Buildings, 43, 2011a, 695-703. doi:10.1016/j.enbuild.2010.11.014 C54, C55, C58

[10] S. C. Saha, Scaling of free convection heat transfer in a triangular cavity for $\operatorname{Pr}>$ 1, Energy and Buildings, 43, 2011b, 2908-2917. doi:10.1016/j.enbuild.2011.07.016 C54, C55, C58

[11] G. A. Holtzman, R. W. Hill, K. S. Bal, Laminar natural convection in isosceles triangular enclosures heated from below and symmetrically cooled from above, J. Heat Transfer, 122, 2000, 485-491. doi:10.1115/1.1288707 C55, C62, C65

[12] S. C. Saha, J. C. Patterson, C. Lei, Natural convection and heat transfer in attics subject to periodic thermal forcing, Int. J. Thermal Sciences, 49, 2010, 1899-1910. doi:10.1016/j.ijthermalsci.2010.05.010 C55, C65

\section{Author addresses}

1. Suvash C. Saha, School of Chemistry, Physics and Mechanical Engineering, Queensland University of Technology, Brisbane, Queensland 4001, Australia. mailto:suvash.saha@@qut. edu. au

2. Y. T. Gu, School of Chemistry, Physics and Mechanical Engineering, Queensland University of Technology, Brisbane, Queensland 4001, Australia. mailto:yuantong.gu@qut.edu.au 